Received: 25.12 .2017

Revised: 26.01 .2018

Accepted: 16.02 .2018

DOI: $10.17804 / 2410-9908.2018 .1 .041-049$

\title{
SUBSTANTIATION OF TECHNOLOGICAL OPERATIONS OF WELDING A SADDLE BRANCH TO POLYETHYLENE PIPES AT TEMPERATURES BELOW AVAILABLE
}

\author{
N. P. Starostin ${ }^{1, \text { a) }}$, M. A. Vasilieva ${ }^{1, b)^{*}}$ \\ Institute of Oil and Gas Problems, Siberian Branch of the Russian Academy of Sciences, \\ 1 Oktyabrskaya St., Yakutsk, 677891, Russian Federation \\ a) (D) https://orcid.org/0000-0002-5686-1817; ${ }^{\text {b) }}$ id http://orcid.org/0000-0002-1805-1776, 困 eowa@ mail.ru
}

*Corresponding author. E-mail: eowa@mail.ru

Address for correspondence: ul. Avtodorozhnaya, 20, 677007, Yakutsk, Russian Federation Tel.: +7(4112) 357293

\begin{abstract}
Additional branches for gas supply systems are generally installed during the completion of the construction of a gas pipeline, often falling in the autumn or winter time, when the air temperature is below the maximum allowable for welding polyethylene pipes. At ambient temperatures below minus $15^{\circ} \mathrm{C}$, it is recommended to weld polyethylene pipes under a shelter, where the temperature is maintained in the permissible temperature range.

The paper proposes a mathematical model for the thermal process of welding a saddle branch to a polyethylene pipe taking into account the heat of phase transition. The corresponding threedimensional non-linear heat equation is solved by the finite element method. As an example, calculations were made for a PE 100 SDR 11 polyethylene pipe and a 100/63 saddle tap. Comparison of the calculated and experimental temperature data establishes the adequacy of the proposed mathematical model to the actual thermal process of welding a saddle branch to a polyethylene pipe.

To obtain a high-quality welded joint, it is necessary to ensure that the temperature field changes according to a certain regularity in the heat-affected zone. Admissible regularity is ensured when welding is performed in the range of climatic air temperatures of above minus $15^{\circ} \mathrm{C}$. In this study, examples are considered for the definition of simple technological operations that ensure such regularity at ambient temperatures below normative ones. For comparison, the dynamics of temperature fields at ambient temperatures of $20^{\circ} \mathrm{C}$ and minus $40^{\circ} \mathrm{C}$ was calculated. Technological operations necessary to weld a saddle branch at the temperatures below the standard are determined from studying the dynamics of temperature profile during the welding of the saddle branch to a polyethylene pipe at various ambient temperatures.
\end{abstract}

Keywords: mathematical model, finite element method, temperature, calculation, thermal process, welding, phase change, crystallization.

\section{References}

1. SP 42-103-2003. Proektirovanie i stroitelstvo gazoprovodov iz polietilenovykh trub i rekonstruktsiya iznoshennykh gazoprovodov [Design and Construction of Polyethylene Gas Pipelines and Reconstruction of Decrepit Gas Pipelines: Handbook of Instructions]. (In Russian).

2. Starostin N.P., Ammosova O.A. Simulation of the Thermal Process of Butt Welding of Polyethylene Pipes at Low Temperatures. J. Eng. Phys. Thermophys., 2016, vol. 89, no. 3, pp. 714-720. DOI: 10.1007/s10891-016-1430-8 
3. Strang G. and Fix G.J. An Analysis of the Finite Element Method, Prentice-Hall, Englewood Cliffs, NJ, 1973.

4. $\quad$ Segerlind L.J. Applied Finite Element Analysis, 2nd edition, Wiley, New York, 1984, 427 p.

5. Chebbo Z, Vincent M., Boujlal A., Gueugnaut D., Tillier Y. Numerical and Experimental Study of the Electrofusion Welding Process of Polyethylene Pipes. Polymer Engineering \& Science, 2015, vol. 55, iss. 1, pp. 123-131. DOI: 10.1002/pen.23878

6. Logg F., Mardal K.-A., Wells G.N., editors. Automated Solution of Partial Differential Equations by Finite Element Method: The FEniCS Book (Lecture Notes in Computational Science and Engineering), Springer, 2012, 731 p.

7. Software package FEniCS. Available at: http://fenicsproject.org/

8. Software package GMSH. Available at: http://geuz.org/gmsh/

9. Samarsky A.A., Vabishchevich P.N. Vychislitelnaya teploperedacha [Computational Heat Transfer]. Moscow, Librokom Publ., 2014, 784 p. (In Russian).

10. Ciarlet Ph.G. The Finite Element Method for Elliptic Problems, North-Holland Publishing Co., Amsterdam and New York, 1978, xvii-530 p. 
Подана в журнал: 25.12.2017

УДК 621.791.461:678.029.43

DOI: $10.17804 / 2410-9908.2018 .1 .041-049$

\title{
ОБОСНОВАНИЕ ТЕХНОЛОГИЧЕСКИХ ОПЕРАЦИЙ ПРИВАРКИ СЕДЛОВЫХ ОТВОДОВ К ПОЛИЭТИЛЕНОВЫМ ТРУБАМ ПРИ ТЕМПЕРАТУРАХ НИЖЕ ДОПУСТИМЫХ
}

\author{
Н. П. Старостин ${ }^{\text {a) }}$, М. А. Васильева ${ }^{\text {()* }}$ \\ Федеральное государственное бюджетное учреждение науки Институт проблем нефти и газа \\ Сибирского отделения Российской академии наук, \\ ул. Октябрьская, 1, Якутск, Российская Федераџия \\ a) iD https://orcid.org/0000-0002-5686-1817; ${ }^{6)}$ iD http://orcid.org/0000-0002-1805-1776, ख eowa@ mail.ru \\ *Ответственный автор. Электронная почта: eowa@mail.ru \\ Адрес для переписки: 677007, г. Якутск, ул. Автодорожная, 20, Российская Федерация \\ Тел.: +7 (4112) 35-72-93
}

Работы по монтажу дополнительных ответвлений для систем газоснабжения обычно проводят в период завершения строительства газопровода, часто попадающего на осеннезимнее время, когда температура воздуха ниже предельно допустимой для проведения сварки полиэтиленовых труб. При температурах окружающего воздуха ниже $-15{ }^{\circ} \mathrm{C}$ сварку полиэтиленовых труб рекомендуется проводить под укрытием, в котором поддерживается температура из допустимого температурного интервала.

В статье предложена математическая модель теплового процесса приварки седлового отвода к полиэтиленовой трубе с учетом теплоты фазового перехода. Соответствующее трехмерное нелинейное уравнение теплопроводности решают методом конечных элементов. В качестве примера расчеты были сделаны для полиэтиленовой трубы ПЭ 100 SDR 11 и седлового отвода 100/63. Сопоставлением расчетных и экспериментальных температурных данных установлена адекватность предложенной математической модели реальному тепловому процессу приварки седлового отвода к полиэтиленовой трубе.

Для получения качественного сварного соединения необходимо обеспечить в зоне термического влияния изменение температурного поля по определенной закономерности. Допустимая закономерность обеспечивается при сварке в диапазоне климатических температур воздуха выше $-15^{\circ} \mathrm{C}$. В статье рассматриваются примеры для определения простых технологических операций, обеспечивающих такую закономерность при температурах окружающего воздуха ниже нормативных. Для сравнения были рассмотрены расчеты динамики температурных полей при температурах окружающего воздуха $20^{\circ} \mathrm{C}$ и $-40{ }^{\circ} \mathrm{C}$. На основе исследования динамики температурного поля при приварке седлового отвода к полиэтиленовой трубе при различных температурах окружающего воздуха определены технологические операции, необходимые для проведения приварки седлового отвода при температурах воздуха ниже нормативного.

Ключевые слова: математическая модель, метод конечных элементов, температура, расчет, тепловой процесс, приварка, фазовый переход, кристаллизация. 


\section{1. Введение}

Седловые отводы с закладным нагревателем широко используются при строительстве систем газоснабжения для монтажа ответвлений от магистральных и распределительных газопроводов из полиэтиленовых труб к промышленным и бытовым потребителям. Сварку полиэтиленовых труб при помощи соединительных деталей с закладными нагревателями следует производить при температуре окружающего воздуха не ниже $-15{ }^{\circ} \mathrm{C}$ [1]. При более низких температурах окружающего воздуха сварочные работы выполняют в укрытии, в котором поддерживается температура из допустимого температурного интервала. Для разработки технологии приварки седлового отвода к полиэтиленовой трубе актуальным является математическое моделирование теплового процесса в сварном соединении с учетом теплоты фазового перехода при оплавлении и кристаллизации полиэтилена и исследование влияния температуры ОВ на динамику температурного поля.

Ранее способ стыковой сварки полиэтиленовых труб на открытом воздухе при температурах ниже нормативных на основе управления теплового процесса сварки была рассмотрена в работе [2]. Рассматриваемое уравнение теплопроводности решалась методом конечных разностей, эффективным для решения задач в областях простой формы.

При моделировании теплового процесса приварки седлового отвода к полиэтиленовой трубы необходимо решение уравнения теплопроводности в области сложной формы. Кроме того, сложную форму имеет закладной нагреватель, представляющий собой металлическую проволоку, интегрированную в седловой отвод. При подсоединении закладного нагревателя к электрическому источнику производится нагрев и оплавление материалов седлового отвода и трубы. Проволока закладного нагревателя расположена внутри седлового отвода по линиям, проекция которых на внутреннюю поверхность отвода представляет окружность или квадрат в зависимости от исполнения. Эффективным методом решения задачи теплопроводности в областях сложной формы является метод конечных элементов $[3,4]$. Численное решение трехмерной задачи определения нестационарного температурного поля при сварке полиэтиленовых труб с помощью муфты с закладным нагревателем методом конечных элементов произведено в работе [5]. При этом для упрощения формы области вместо круглой трубы и муфты рассматривалась трехмерная задача определения температурного режима сварки двух плоских пластин, между которыми проложен закладной нагреватель.

В работе на основе исследования динамики температурного поля при приварке седлового отвода к полиэтиленовой трубе при различных температурах окружающего воздуха определены технологические операции, необходимые для проведения приварки седлового отвода при температурах воздуха ниже нормативного.

\section{2. Математическая постановка задачи}

Рассмотрим расчетную схему (рис. 1) приварки седлового отвода 1 (область $D_{1}$ ) к участку полиэтиленовой трубы 2 (область $D_{2}$ ). Нагрев и оплавление свариваемых поверхностей производится закладным нагревательным элементом 3 (область $D_{3}$ ). Нагревательный элемент для упрощения геометрии расчетной области и уменьшения времени расчета примем в виде кольца. Границы промежуточной фазы между твердым и жидким веществом определяются температурами солидуса $T_{S}$ и ликвидуса $T_{L}$. Температурное поле в сварном соединении определяется из уравнения теплопроводности:

$$
\tilde{C} \rho_{i} \frac{\partial T}{\partial t}=\operatorname{div}\left(\lambda_{i} \operatorname{grad} T\right)+\varepsilon Q(r, z), x \in D, 0<t \leq t_{m},
$$

For citation: Starostin N.P., Vasilieva M.A. Substantiation of technological operations of welding a saddle branch to polyethylene pipes at temperatures below available // Diagnostics, Resource and Mechanics of materials and structures. - 2018. - Iss. 1. - P. 41-49. - DOI: 10.17804/2410-9908.2018.1.041-049. 
где $T$ - температура; $\rho$ - плотность материала; $\tilde{C}$ - удельная теплоемкость; $\lambda$ - коэффициент теплопроводности; $\varepsilon=\left\{\begin{array}{l}1, x \in D_{3} \\ 0, x \in D_{1} \cup D_{2}\end{array} ; Q(t)-\right.$ плотность теплового источника, $D=\bigcup_{i=1}^{3} D_{i}$

$$
Q(t)=\left\{\begin{array}{l}
\frac{U^{2}}{R(1+\beta(T(x)-20)) V}, x \in D_{3} \\
0, x \in D_{1} \cup D_{2}
\end{array},\right.
$$

где $U$ - напряжение, подаваемое на нагревательный элемент; $R$ - сопротивление; $\beta$ - температурный коэффициент сопротивления; $V$ - объем нагревательного элемента.

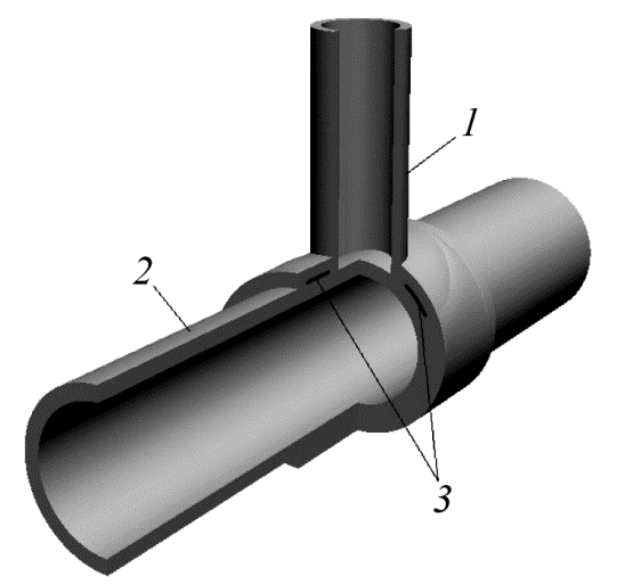

Рис. 1. Расчетная схема: 1 - седловой отвод; 2 - труба; 3 - нагревательный элемент

На свободных поверхностях трубы и седлового отвода Г задается условие конвективного теплообмена:

$$
-\left.\lambda \frac{\partial T}{\partial n}\right|_{\Gamma}=\alpha\left(T-T_{0}\right)
$$

где $\alpha$ - коэффициент теплообмена; $T_{0}$ - температура окружающего воздуха. На торцах трубы и отвода $\Gamma_{T}$ задается граничное условие первого рода:

$$
\left.T\right|_{\Gamma_{T}}=T_{0}
$$

В начальный момент времени берется равным температуре окружающего воздуха:

$$
T(x, 0)=T_{0} .
$$

В математической модели для описания теплового процесса в полимерах промежуточную фазу между твердым и жидким веществом, в которой вещество находится как в твердом, так и в жидком состоянии учитывается в коэффициенте теплоемкости по формуле [2]: 


$$
\tilde{C}(T)=\left\{\begin{array}{l}
C_{1}, \quad T \leq T_{S} \\
C_{1}+\frac{q(T)}{\Delta}, \quad T_{S}<T<T_{L}, \quad \Delta=\frac{\left(T_{L}-T_{S}\right)}{\left(t_{2}-t_{1}\right)}, \\
C_{2}, \quad T \geq T_{L}
\end{array}\right.
$$

где $q(T)$ - зависимость теплового потока от температуры, отнесенная к единице массы вещества, регистрируемая дифференциальным сканирующим калориметром (ДСК); $\Delta$ - скорость нагрева, варьируемая в ДСК.

\section{3. Результаты расчета}

Для вычислительной реализации модельной задачи использовали программный пакет свободного доступа FEniCS [6,7]. Геометрию области строили с использованием программы GMSH [8]. Триангуляцию построенной области производили автоматически также средствами GMSH.

Аппроксимация по времени проводилась с использованием неявной схемы [9]. Для решения задачи (1)-(4) методом конечных элементов рассматривается вариационная задача нахождения элемента $T$ [10]:

$$
a(T, v)=f(v), T \in D, v \in V,
$$

где $a(T, v)=\frac{1}{\tau} \int_{D} \rho \tilde{C}\left(T^{n}\right) T^{n+1} v d x+\int_{D}\left(\lambda\left(T^{n}\right) \operatorname{grad} T^{n+1}, \operatorname{grad} v\right) d x+\int_{\Gamma} \alpha T v d \gamma$

$f(v)=\frac{1}{\tau} \int_{D} \rho \tilde{C}\left(T^{n}\right) T^{n} v d x+\int_{D} Q\left(t^{n}\right) v d x+\int_{\Gamma} \alpha T_{0} v d \gamma$

Для получения качественного сварного соединения необходимо обеспечить в зоне термического влияния изменение температурного поля по определенной (допустимой) закономерности, при которой формируется структура, обусловливающая необходимую прочность соединения. Считается, что допустимая закономерность обеспечивается при сварке в диапазоне климатических температур воздуха выше $-15^{\circ} \mathrm{C}$. Задача состоит в определении простых технологических операций, выполнением которых возможно обеспечить такую закономерность изменения температурного поля в зоне термического влияния при температурах окружающего воздуха ниже нормативных. Для определенности в качестве допустимой закономерности будем рассматривать динамику температурного поля сварки при температуpe $\mathrm{OB} 20^{\circ} \mathrm{C}$.

На рис. 2 представлено сравнение размеров оплавленной зоны (ограниченной изотермой со значением температуры $130{ }^{\circ} \mathrm{C}$ ) в сечении вертикальной плоскостью при сварке при различных температурах ОВ в момент завершения нагрева согласно существующего режима приварки седлового отвода 110/63 к полиэтиленовой трубе ПЭ100 диаметром 110, SDR 11 (подаваемое в нагревательный элемент напряжение 39,5 В, время нагрева 210 с). Меньший размер оплавленной зоны при низкой температуре ОВ свидетельствует о меньшем объеме полученного расплава, следствием которого будет недостаточное давление свариваемых поверхностей, более интенсивная кристаллизация и формирование мелкой структуры материала сварного шва и пр., приводящие к низкому значению прочности соединения. Результаты расчета показывают необходимость предварительного подогрева при сварке в условиях низких температур.

For citation: Starostin N.P., Vasilieva M.A. Substantiation of technological operations of welding a saddle branch to polyethylene pipes at temperatures below available // Diagnostics, Resource and Mechanics of materials and structures. - 2018. - Iss. 1. - P. 41-49. - DOI: 10.17804/2410-9908.2018.1.041-049. 
Вычислительными экспериментами покажем, что предварительный подогрев без обеспечения необходимых условий охлаждения также не приводит к формированию качественного сварного шва. Рассмотрим тепловой процесс приварки седлового отвода к трубе, выполняемого при температуре ОВ ниже нормативной. Пусть при использовании предварительного подогрева в зоне термического влияния в конце нагрева (оплавления) получено распределение температуры, характерное при приварке при температуре ОВ $20{ }^{\circ} \mathrm{C}$. Предположим, что далее охлаждение сварного соединения проводилось на открытом воздухе при температуре ниже нормативной. На рис. 3 представлено сравнение временных зависимостей максимальных температур при приварке седлового отвода к трубе при температуре $-40{ }^{\circ} \mathrm{C}$ по описанному режиму и при допустимых для сварки при температурах 20 и $-15{ }^{\circ} \mathrm{C}$ по регламентированному режиму. Аналогичные зависимости получаются и для других точек соединения.

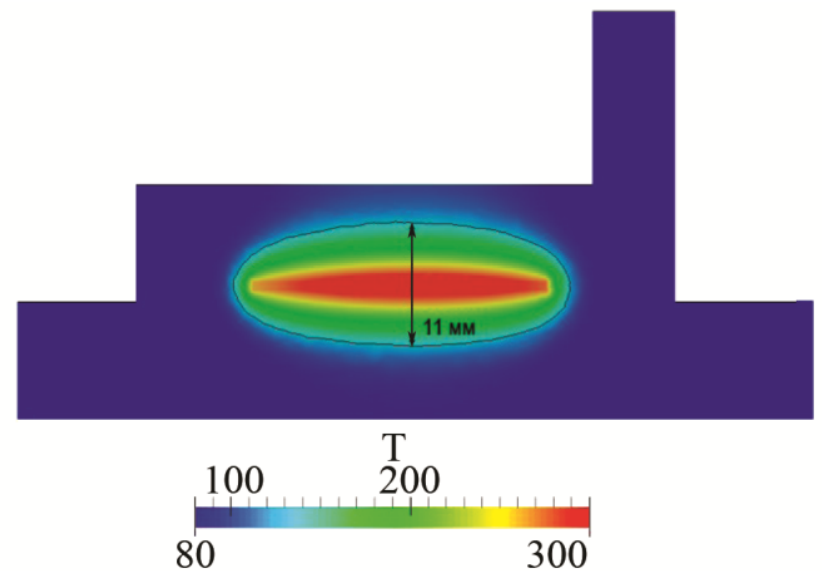

$a$

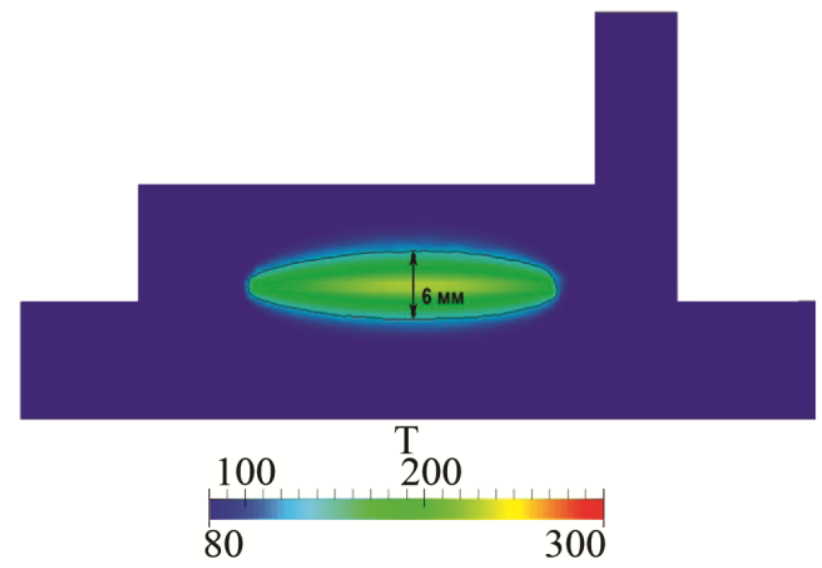

6

Рис. 2. Изотермы со значением температуры $130{ }^{\circ} \mathrm{C}$ в сечении седлового отвода и трубы вертикальной плоскостью в момент завершения нагрева $(t=210 \mathrm{c})$ при температуре $\mathrm{OB}: a-20{ }^{\circ} \mathrm{C} ; \sigma--40^{\circ} \mathrm{C}$

Результаты расчетов показывают, что при приварке в условиях низких температур обеспечение такого же распределения температуры в сварном соединении, как и при допустимой для сварки температуре, и охлаждение соединения на открытом воздухе при низкой температуре, приводит к заметному увеличению скорости охлаждения. Для снижения темпа охлаждения необходимо использование теплоизоляции. 


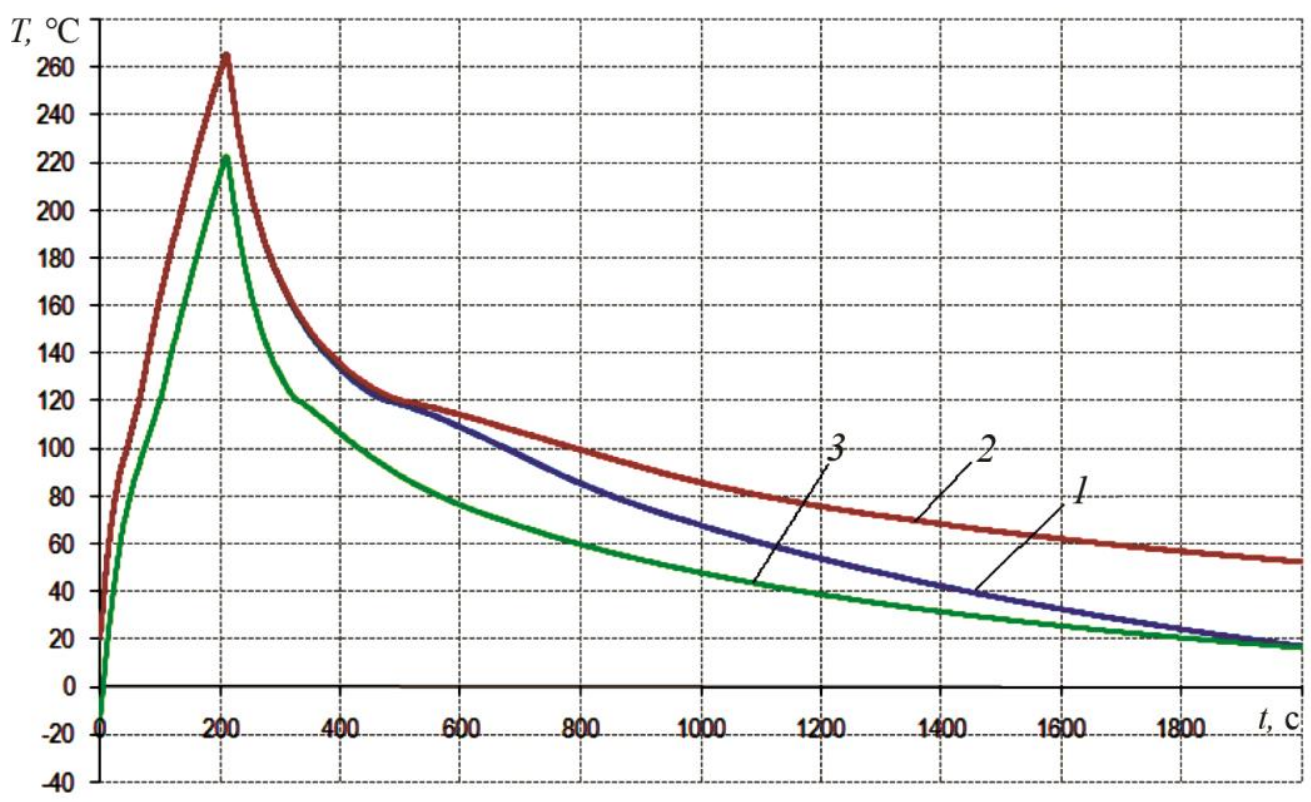

Рис. 3. Временные зависимости максимальных температур при различных режимах приварки седлового отвода к полиэтиленовой трубе: 1 - нагрев при температуре $\mathrm{OB} 20{ }^{\circ} \mathrm{C}$ и охлаждение при $-40{ }^{\circ} \mathrm{C} ; 2$ - нагрев и охлаждение при температуре $\mathrm{OB} 20{ }^{\circ} \mathrm{C}$; 3 - нагрев и охлаждение при температуре $\mathrm{OB}-15^{\circ} \mathrm{C}$

\section{4. Заключение}

Предложена математическая модель теплового процесса приварки седлового отвода к полиэтиленовой трубе, учитывающая поглощение (при оплавлении) и выделение (при кристаллизации) теплоты в интервале температур.

На основе анализа динамики температурных полей при приварке седлового отвода к полиэтиленовой трубе при температурах ОВ ниже нормативных показана необходимость проведения предварительного подогрева до допустимой для сварки температуры и охлаждения сварного соединения под слоем теплоизоляции.

\section{Литература}

1. СП 42-103-2003. Проектирование и строительство газопроводов из полиэтиленовых труб и реконструкция изношенных газопроводов.

2. Starostin N. P., Ammosova O. A. Simulation of the Thermal Process of Butt Welding of Polyethylene Pipes at Low Temperatures // J. Eng. Phys. Thermophys. - 2016. - Vol. 89, no. 3. P. 714-720. - DOI: 10.1007/s10891-016-1430-8

3. Стренг Г., Фикс Дж. Теория метода конечных элементов / пер. с англ. В. И. Агошкова, В. А. Василенко, В. В. Шайдурова ; под ред. Г. И. Марчука. - М. : Мир, 1977. - 349 с.

4. Сегерлинд Л. Применение метода конечных элементов / пер. с англ. - М. : Мир, 1979. - 392 c.

5. Numerical and Experimental Study of the Electrofusion Welding Process of Polyethylene Pipes / Z. Chebbo, M. Vincent, A. Boujlal, D. Gueugnaut, Y. Tillier // Polymer Engineering and Science. - 2015. - Vol. 55, iss. 1. - P. 123-131. - DOI: 10.1002/pen.23878

6. Automated Solution of Partial Differential Equations by Finite Element Method: The FEniCS Book / ed. by F. Logg, K.-A. Mardal, G. N. Wells. - Springer, 2012. - 731 p.

7. Software package FEniCS. - URL: http://fenicsproject.org/

8. Software package GMSH. - URL: http://geuz.org/gmsh/ 
9. Самарский А. А., Вабищевич П. Н. Вычислительная теплопередача. - М. : ЛИБРОКОМ, 2014. - 784 c.

10. Сьярле Ф. Метод конечных элементов для эллиптических задач. - М. : Мир, 1980. $512 \mathrm{c}$. 